\title{
High y DIS Cross Section Measurement with H1
}

\author{
Nataša Raičević \\ on behalf of the H1 Collaboration
}

University of Montenegro - Faculty of Science

Cetinjski put BB, 81000 Podgorica - Montenegro

\begin{abstract}
A new preliminary measurement of the inclusive ep scattering cross section from $\mathrm{H} 1$ experiment at HERA is presented in the region of low four-momentum transfer squared, $12 \mathrm{GeV}^{2}<Q^{2}<50 \mathrm{GeV}^{2}$ and very high inelasticity, $0.75<y<0.9$. The cross section in this kinematic domain is sensitive to the longitudinal structure function $F_{L}$ and thus provides additional constraints to the DGLAP evolution. The results are based on data collected in 2003-2006 (HERA-II). About equal luminosities obtained for $\mathrm{e}^{+} \mathrm{p}$ and $\mathrm{e}^{-} \mathrm{p}$ collisions allow for a high precision control of background processes.
\end{abstract}

\section{Introduction}

The $\mathrm{e}^{ \pm} \mathrm{p}$ deep-inelastic scattering (DIS) double-differential cross section at low values of squared four momentum transfer, $Q^{2}$, in the one-photon exchange approximation, can be expressed as,

$$
\frac{d^{2} \sigma}{d x d Q^{2}} \cdot \frac{Q^{4} x}{2 \pi \alpha^{2} Y_{+}}=\sigma_{r}=F_{2}\left(x, Q^{2}\right)-\frac{y^{2}}{Y_{+}} \cdot F_{L}\left(x, Q^{2}\right),
$$

where $x$ is the Bjorken scaling variable, $y=Q^{2} / s x$ is the inelasticity which is a fraction of the electron's ${ }^{\mathrm{a}}$ energy loss, $s$ is the center of mass energy squared of the electron-proton system and $Y_{+}=1+(1-y)^{2}$. The two structure functions $F_{2}$ and $F_{L}$ obey the relation $0 \leq F_{L} \leq F_{2}$ due to the positivity of the cross sections for transversely and longitudinally polarised photons scattering off protons. Therefore, the longitudinal structure function, $F_{L}$, gives a sizable contribution to the cross section only at large values of the inelasticity $y$. The longitudinal structure function, $F_{L}$, is identically zero in lowest order QCD, but due to gluon radiation gets a non-zero value in perturbative QCD. The measurement of $F_{L}$ can thus provide constraints on the gluon density function which are complementary to those obtained from the scaling violations of $F_{2}$ assuming DGLAP evolution[2].

At high $y$ the event kinematics is best determined using the measured energy of the scattered electron, $E_{e}{ }^{\prime}$, and its polar angle, $\theta_{e}$, (electron method) according to the relations

$$
y=1-\frac{E_{e}{ }^{\prime}}{E_{e}} \sin ^{2}\left(\theta_{e} / 2\right), \quad Q^{2}=\frac{E_{e}{ }^{\prime 2} \sin ^{2} \theta_{e}}{1-y} .
$$

Thus, to measure at largest values of $y$, it is necessary to reach as low as possible $E_{e}{ }^{\prime}$.

The new high $y$ cross section measurement from H1 is based on HERA-II data with integrated luminosity of $96 \mathrm{pb}^{-1}$ where $51 \mathrm{pb}^{-1}$ is from $\mathrm{e}^{+} \mathrm{p}$ and $45 \mathrm{pb}^{-1}$ from $\mathrm{e}^{-} \mathrm{p}$ interactions. The kinematic plane covered in this analysis is shown in Figure 1 together with kinematic planes from the analysis of HERA-I data from $\mathrm{H} 1$ and fixed target experiments.

\footnotetext{
${ }^{\text {aT }}$ The name electron in the text is used to denote both electrons and positrons.
} 


\section{Principle of the measurement}

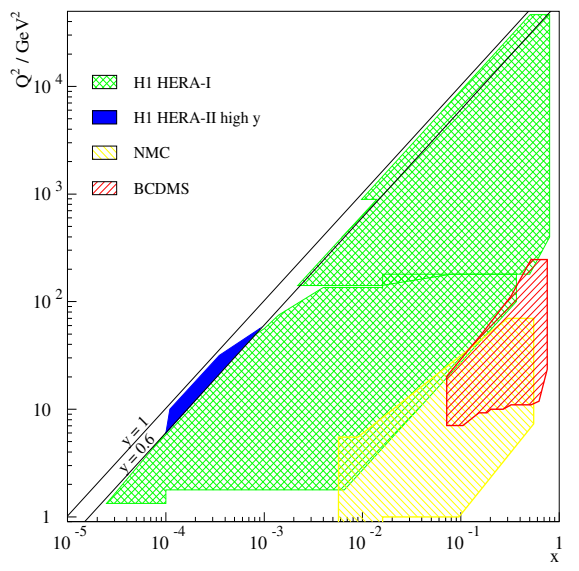

Figure 1: Kinematic plane covered by $\mathrm{H} 1$ and fixed target experiments. Dark region, labeled H1 HERA-II high $y$, corresponds to this analysis.

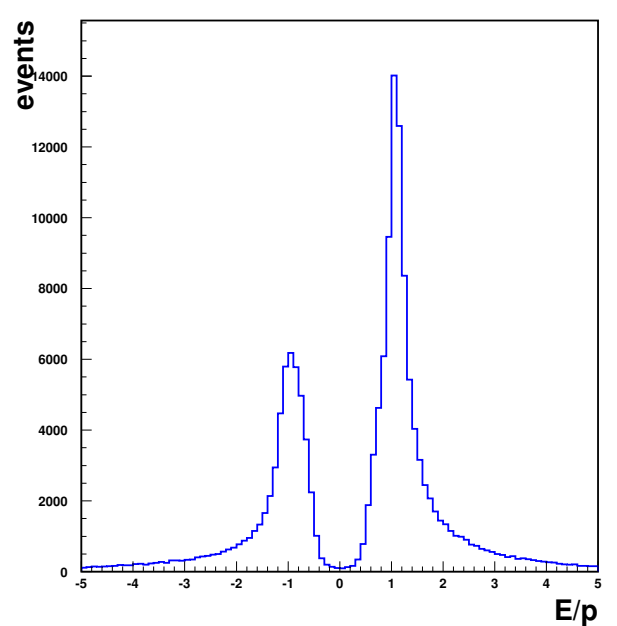

Figure 2: Energy over momentum for tracks from $\mathrm{e}^{+} \mathrm{p}$ interactions linked to clusters in SpaCal with energy from 3.4 to $10 \mathrm{GeV}$.
To select events with low and medium $Q^{2}$ with the $\mathrm{H} 1$ detector [3], at least one cluster in the backward electromagnetic calorimeter (spaghetti calorimeter $\mathrm{SpaCal}$ ) is required. Copious photoproduction processes often lead to electron-like low energy deposits in SpaCal. Thus the high level of the photoproduction background is the main difficulty for a cross section measurement at large values of $y$. A sizable background of hadrons originates also from deep inelastic scattering because at high $y$ and low $Q^{2}$ the hadronic final state is scattered backwards. To suppress hadronic contributions, cuts on the shower shape estimators are performed. An additional suppression of the radiative events and photoproduction background is performed by requiring energy and longitudinal momentum conservation. A well reconstructed vertex is required in the interaction region to further reduce background and contribution from beam background events. The cuts were optimized to avoid efficiency loss as much as possible and to still significantly reduce background.

The high level of the background at large values of $y$ leads directly to an additional uncertainty in the background subtraction procedure. The background also complicates the estimation of the signal selection efficiency in an unbiased way. To achieve an efficient rejection and identification of photoproduction background, this analysis does not rely on the Monte Carlo simulation for the background level estimation, but uses experimental information by employing the charge assignment of central tracks associated with SpaCal energy cluster. The sample of candidates with negative charge is taken to represent the background in the positron data sample and vice versa. This allows the energy range to be extended down to $3.4 \mathrm{GeV}$ corresponding to $y \leq 0.9$.

Figure 2 shows the energy over momentum ratio from $\mathrm{e}^{+} \mathrm{p}$ interactions for tracks which pass all the cuts and are linked to clusters with energy from $3.4 \mathrm{GeV}$ to $10 \mathrm{GeV}$ (energy is measured from SpaCal and momentum from the central tracking chambers). The smaller peak corresponds to tracks with negative charge and is almost a pure background with a charge misidentification probability about $0.5 \%$. These tracks are termed as wrong sign tracks. The higher peak, near $\mathrm{E} / \mathrm{p}=1$, contains signal plus remaining positive background (correct sign tracks) which can be determined from $\mathrm{e}^{-} \mathrm{p}$ interactions. 

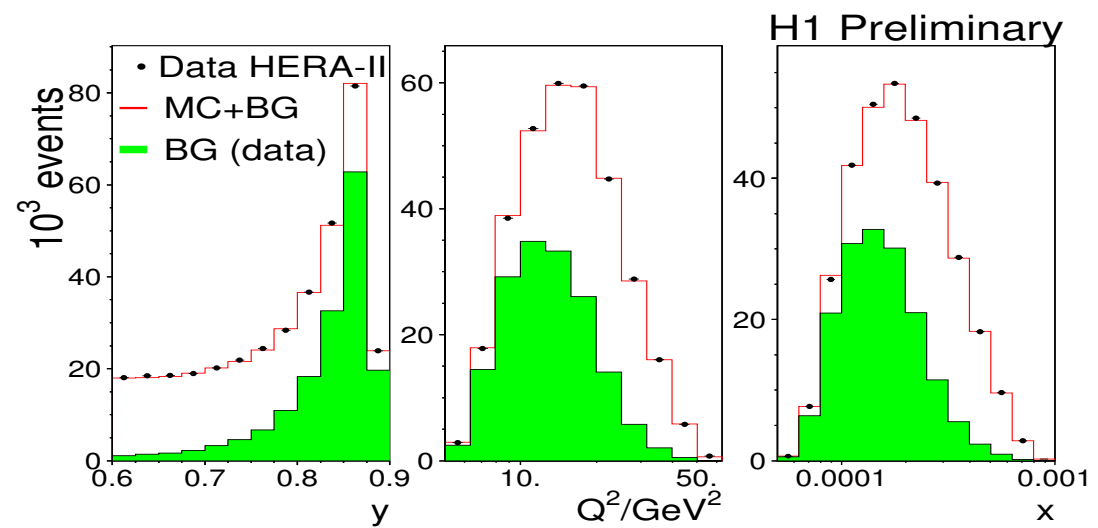

Figure 3: Comparison of the correct sign data (dots) with a sum of background determined from the wrong sign data (shadowed histogram) and DIS Monte Carlo simulation for $y, Q^{2}$ and $x$ distributions.
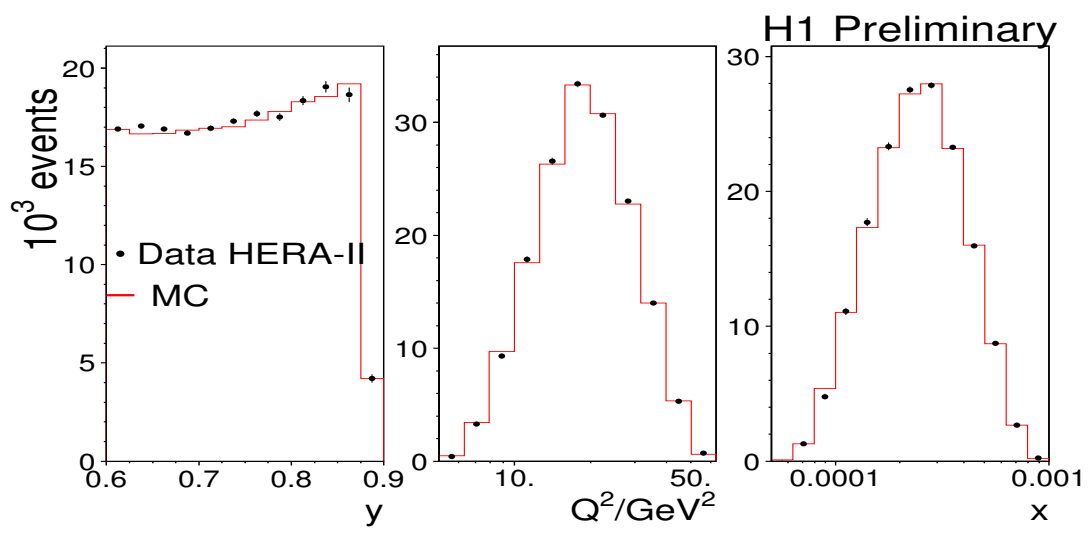

Figure 4: Comparison of the background subtracted data (dots) and DIS Monte Carlo simulation (histogram) for $y, Q^{2}$ and $x$ distributions.

The efficiency of the high $y$ first level trigger used in the analysis is uniform in radius and energy in SpaCal and is about $97 \%$ for the total data sample.

\section{Results}

Figure 3 shows a comparison of the correct sign data (dots) with a sum of background determined from the wrong sign data (shadowed histogram) and DIS Monte Carlo simulation for $y, Q^{2}$ and $x$ distributions. The simulation of the DIS cross section uses a QCD parameterisation of the structure functions which in an iterative process has been adjusted to the measured cross section. A comparison for the corresponding background subtracted distributions is shown in Figure 4. As can be seen from these figures, data are well described by Monte Carlo simulation.

The systematic uncertainty of the new measurement is $2-3 \%$ and is dominated by the track link efficiency uncertainty which is estimated to be $1.5 \%$. Other sources of uncorrelated 


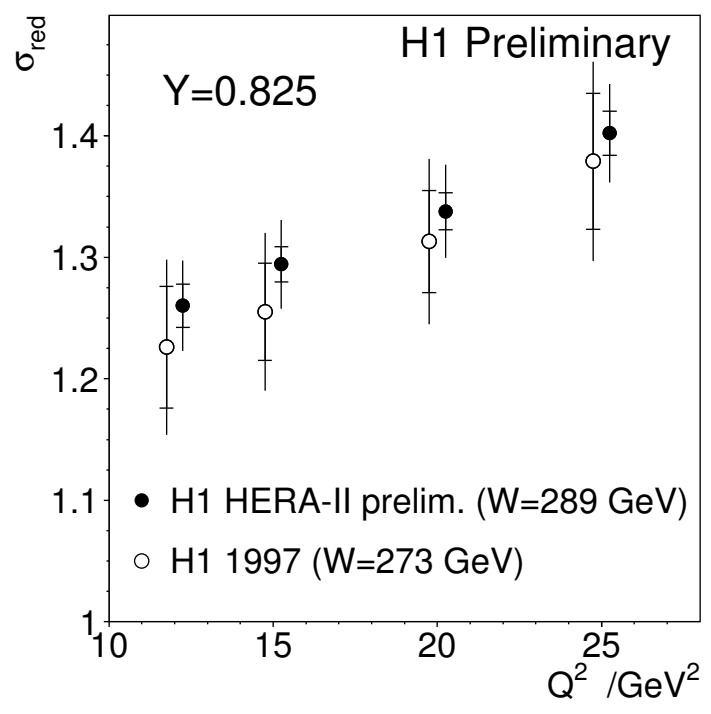

Figure 5: Comparison of $Q^{2}$ dependence of preliminary cross section measurement (solid circles) at inelasticity $y=0.825$ with the published H1 result (open circles). Note that the published data corresponds to a slightly lower center of mass energy.

systematic uncertainties are: trigger efficiency $(1.2 \%)$, track charge identification (0.5\%) and electron identification $(0.5 \%)$. Concerning correlated errors, the main influence comes from the $E_{e}{ }^{\prime}$ energy scale uncertainty $(1 \%$ at $3 \mathrm{GeV})$, the $\theta_{e}$ uncertainty $(1 \mathrm{mrad})$ and the calorimeter hadronic energy scale uncertainty $( \pm 1 \mathrm{GeV})$.

Figure 5 compares the new preliminary cross section depending on $Q^{2}$ at inelasticity $y=0.825$ with the published $\mathrm{H} 1$ result based on HERA-I data $[4,5]$. The new measurement has significantly reduced uncertainties. The total uncertainty is reduced by about factor of two. Unlike for the published H1 result, based on HERA-I data, for this analysis a large sample of data from $\mathrm{e}^{-} \mathrm{p}$ interactions is available for cross section measurement and the control of the charge asymmetry which provides much improved understanding of systematics.

This analysis shows that at $\mathrm{H} 1$ there are advanced tools to cope with a large background at low energies which is vital for the direct accurate measurement of the longitudinal structure function as is expected to be derived from the HERA low proton energy run data.

\section{References}

[1] Slides: http://indico.cern.ch/contributionDisplay $\cdot$ py?contribId=26\&sessionId=8\&conf Id=9499

[2] V. Gribov and L. Lipatov, Sov. J. Nucl. Phys. 15, 438 and 675 (1972); L. Lipatov, Sov. J. Nucl. Phys. 20, 94 (1975); G. Altarelli and G. Parisi, Nucl. Phys. B 126, 298 (1977); Y. Dokshitzer, Sov. Phys. JETP , 641 (1977).

[3] H1 Collaboration, I. Abt et al., Nucl. Instr. and Meth. A386 310 and A386 348 (1997).

[4] A.A. Glazov, PhD Thesis, Berlin, Humboldt-University, 1998, DESY-Thesis-1998-005.

[5] H1 Collaboration, C. Adloff et al., Eur. Phys. J. C19, 269 (2001). 